\title{
MOR for the MSFEM of the Eddy Current Problem in Linear Laminated Media
}

\author{
Hollaus Karl ${ }^{*}$, Schöberl Joachim, Markus Schöbinger \\ Department of Analysis and Scientific Computing, Vienna University of Technology, Wiedner Hauptstraße 8-10, \\ 1040 Vienna, Austria; *karl.hollaus@tuwien.ac.at
}

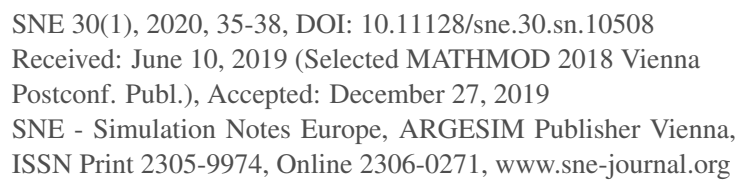

SNE 30(1), 2020, 35-38, DOI: 10.11128/sne.30.sn.10508 Received: June 10, 2019 (Selected MATHMOD 2018 Vienna

Postconf. Publ.), Accepted: December 27, 2019

SNE - Simulation Notes Europe, ARGESIM Publisher Vienna, ISSN Print 2305-9974, Online 2306-0271, www.sne-journal.org

Abstract. The multiscale finite element method has proven to be a powerful method in the simulation of eddy currents in laminated cores. However, the resulting equation systems of the problems relevant in electrical engineering are still too large to be solved conveniently. The use of model order reduction methods to overcome this shortcoming is obvious. The feasibility to exploit the specific approach of the multiscale finite element method in terms of a structural model order reduction method has been studied in this work. A small numerical example shows satisfactory results.

\section{Introduction}

The simulation of the eddy currents in electrical devices with the finite element method (FEM) is satisfactory. However, the large systems to be solved result in high computational costs, i.e. memory requirement and computation time. Although the multiscale finite element method (MSFEM) can be exploited to simulate eddy currents in laminated iron more efficiently the complexity of the problems are still too large to solve them conveniently. The computational costs are a multiple of the costs of anisotropic models in brute force methods according to the components used in the multiscale formulation, compare with [2].

Model order reduction (MOR) has proven to be a powerful methodology to reduce the costs and is well established for linear problems. MOR with proper orthogonal decomposition (POD) has been applied to solve large scale linear problems in computational electromagnetics very successful.
Strategies to select an optimal number of snapshots except those with the largest singular values can be found in [5] and [3]. Those MOR methods are interesting which exploit properties of specific problems. Splitting of the domain into a region where the solution changes strongly due to a parameter variation and the rest, MOR is applied to the rest with almost constant solution in [4]. For example, the speedup factor is about 1.6 for quasitatic problems in 2D by MOR with POD applied only to the linear domain in [6]. MOR is frequently used to facilitate the simulation of electrical machines, see for example [1]. A simple MOR method has been presented in [8] in the context of time stepping for nonlinear 3D problems and a method based on a mixed formulation [9], which can be easily implemented in an existing code for the mixed formulation and standard FEM.

In the present work, the idea is to exploit the specific structure of systems coming from the MSFEM for the eddy current problem (ECP) in laminated media for MOR. For example, the entire problem region can be subdivided into air and the laminated media on the one hand and, on the other, the total solution is composed of a large scale and fine scale part. This work focuses on the second aspect which will be called structural model order reduction (SMOR), see also [3, 7].

The aim is to study the feasibility to exploit the structure of specific systems arising out of MSFEM of ECPs with laminated media for MOR. Much more accurate results are expected by MSFEM with MOR than by FEM with MOR with the same effort.

First, the basic ECP studied in the present work uses a single component current vector potential (SCCVP) $T$ and is discussed in Section 2. Then, MSFEM for $T$ is introduced. Next, MOR and structural model order reduction (SMOR) are explained briefly in Section 3. A comparison of numerical results obtained by MOR and SMOR are presented in Section 4. 


\section{Higher Order MSFEM with the Single Component Current Vector Potential $T$}

\subsection{Boundary Value Problem with $T$}

A current vector potential $T$ can be introduced by $J=$ $\operatorname{curl} T$ fulfilling $\operatorname{div} J=0$ exactly. This work deals with the single component current vector potential $T$, e.g., pointing in $z$-direction $T=T e_{z}$ in the frequency domain. A simple boundary value problem (BVP) of the ECP in the frequency domain reads, see Figure 1:

$$
\begin{aligned}
\operatorname{curl} \frac{1}{\sigma} \operatorname{curl} T+j \mu \omega T & =0 \text { in } \Omega \subset \mathbb{R}^{2} \\
T & =T_{0} \text { on } \Gamma
\end{aligned}
$$

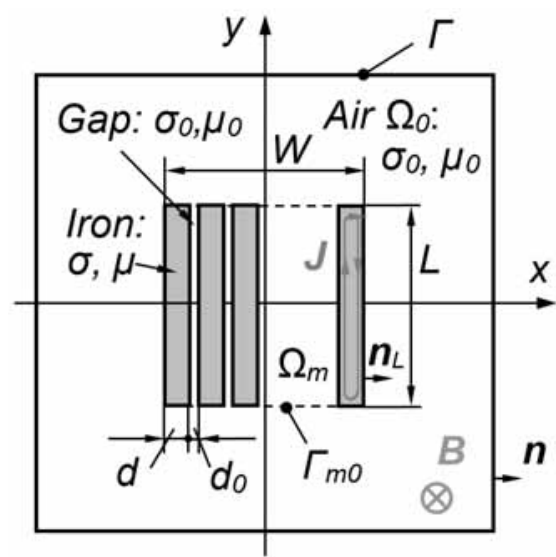

Figure 1: Eddy current problem in 2D.

\subsection{Weak Form with $T$}

The weak form for the FEM in the frequency domain reads:

Find $T_{h} \in V_{h, T_{0}}:=\left\{T_{h} \in \mathscr{U}_{h}: T_{h}=T_{0}\right.$ on $\left.\Gamma\right\}$, such that

$$
\int_{\Omega} \frac{1}{\sigma} \operatorname{curl} T_{h} \cdot \operatorname{curl} t_{h} d \Omega+j \omega \int_{\Omega} \mu T_{h} t_{h} d \Omega=0
$$

for all $t_{h} \in V_{h, 0}$, where $\mathscr{U}_{h} \subset H^{1}(\Omega)$.

\subsection{Higher Order Multiscale Finite Element Method with $T$}

The multiscale approach up to the order 4 for the single component current vector potential

$$
\tilde{T}(x, y)=T_{0}(x, y)+\phi_{2}(x) T_{2}(x, y)+\phi_{4}(x) T_{4}(x, y)
$$

is considered with even micro-shape functions $\phi_{2}$ and $\phi_{4}$ shown in Figure 2. Simply speaking $T$ corresponds to the magnetic field strength $H$ which is an even function in the lamination, therefore the micro-shape functions $\phi_{2}$ and $\phi_{4}$ are used in (4).

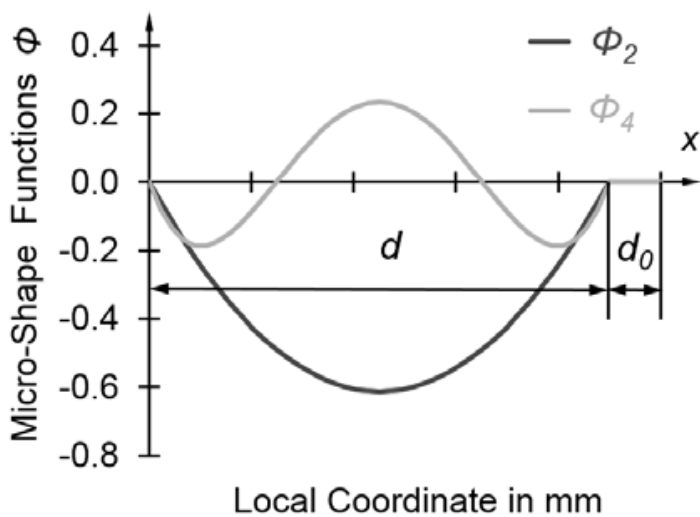

Figure 2: Even micro-shape functions $\phi_{2}$ and $\phi_{4}$.

\subsection{Weak Form of MSFEM with $T$}

The weak form reads as:

Find $\left(T_{0 h}, T_{2 h}, T_{4 h}\right) \in V_{h, T_{0}}:=\left\{\left(T_{0 h}, T_{2 h}, T_{4 h}\right): T_{0 h} \in\right.$ $\mathscr{U}_{h}, T_{2 h}$ and $T_{4 h} \in \mathscr{V}_{h}, T_{0 h}=T_{0}$ on $\Gamma$ and $T_{2 h}=0$ and $T_{4 h}=0$ on $\left.\Gamma_{m 0,1} \subset \Gamma_{m 0}\right\}$, such that

$$
\int_{\Omega} \frac{1}{\sigma} \operatorname{curl} \tilde{T}_{h} \cdot \operatorname{curl} \tilde{t}_{h} d \Omega+j \omega \int_{\Omega} \mu \tilde{T}_{h} \tilde{t}_{h} d \Omega=0
$$

for all $\left(t_{0 h}, t_{2 h}, t_{4 h}\right) \in V_{h, 0}$, where $\mathscr{U}_{h}$ is a subspace of $H^{1}(\Omega), \mathscr{V}_{h}$ of $H^{1}\left(\Omega_{m}\right)$ and $\phi_{2}$ and $\phi_{4} \in H_{p e r}^{1}\left(\Omega_{m}\right)$.

\section{MOR and SMOR}

Assume that the MSFEM (5) results in the linear equation system

$$
A x=f .
$$


Furthermore, $m$ snapshots $x_{i}$, i.e. solutions of $A_{i} x_{i}=f$ by modifying a parameter are calculated and inserted as column vectors in the snapshot matrix $S$ with dimension $n \times m$, where usually $n \gg m$ holds. The present work uses the relative permittivity $\mu_{r}$ as parameter. Next, for the POD based MOR a singular value decomposition (SVD)

$$
S=U \Sigma V^{*}
$$

the star marks conjugate transpose of $V$, is carried out. Matrices $U(n \times n)$ and $V(m \times m)$ are Hermitian matrices. The singular values $\sigma_{i}$ are arranged in the diagonal of $\Sigma$ with $\sigma_{i} \geq \sigma_{i+1}$. Now, an appropriate reduced basis

$$
W=\left[u_{1} \sigma_{1}, u_{2} \sigma_{2}, \ldots, u_{r} \sigma_{r}\right]
$$

matrix $W$ represents the projection matrix, is selected considering the essential singular values $\sigma_{i}$, where $r \geq$ $m$ is valid. With $x=W y$ the reduced order model

$$
W^{T} A W y=W^{T} f=K y=g
$$

is obtained. Similarly, SVDs are carried out of all partitions $S_{i}$, where $S=\left(S_{0}, S_{2}, S_{4}\right)^{T}$, according to the unknowns $T_{0}, T_{2}$ and $T_{4}$ in the approach (4). Therefore, SMOR yields a larger reduced model order than MOR.

\section{Numerical Results}

The model shown in Figure 1 consists of 10 laminates, $d=1.8 \mathrm{~mm}$, and air gaps in between, $d_{0}=0.2 \mathrm{~mm}$. The dimensions of the domains are $\left|\Omega_{m}\right|=20 \times 20 \mathrm{~mm}^{2}$ and $|\Omega|=40 \times 40 \mathrm{~mm}^{2}$. The frequency $f$ was chosen with $50 \mathrm{~Hz}$ and the conductivity $\sigma$ with $2 \cdot 10^{6} \mathrm{~S} / \mathrm{m}$.

The relative error presented in Figure 3 is defined by comparing the eddy current losses $P$ obtained by MOR or SMOR with those of MSFEM:

$$
\text { Relative error in } \%=\frac{P_{(S) M O R}-P_{M S F E M}}{P_{M S F E M}} \cdot 100
$$

For the snapshots, $\mu_{r}$ has been selected with 125,625 , 3125,15625 and 78125. The solutions in Figure 3 are calculated at $\mu_{r}$ equals $375,1875,9375$ and 46875 , i.e. $m=5$. The number of basis vectors used in the reduced basis is denoted by $k$. SMOR provides already for a very small reduced basis reasonable results. The error of MOR decreases for increasing $\mu_{r}$ clearly. MOR and SMOR reduce the MSFEM system by factor of about 100.

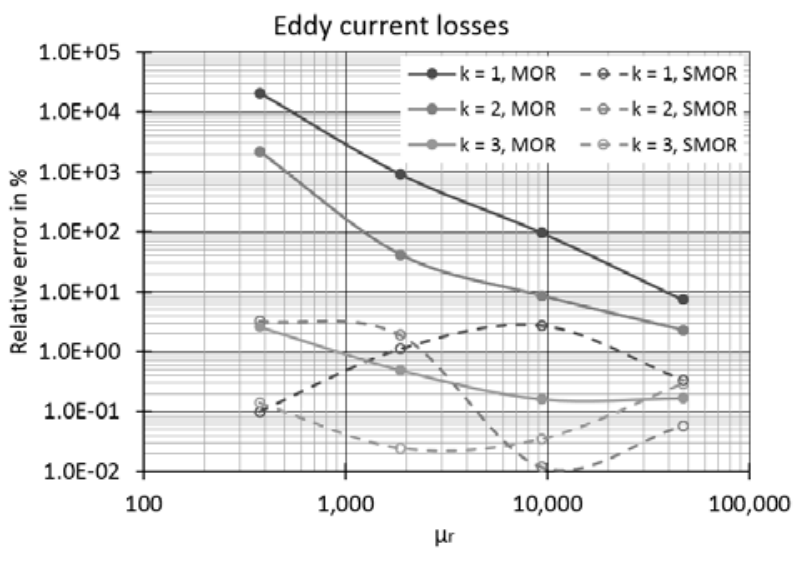

Figure 3: Comparison of model order reduction (MOR) with structural model order reduction (SMOR).

\section{Conclusion}

SMOR seams to be working properly already with very few basis vectors, i.e. low dimension of the reduced basis. An extension of SMOR to large and nonlinear problems in $3 \mathrm{D}$ will be studied in the future.

\section{Acknowledgement}

This work was supported by the Austrian Science Fund (FWF) under Project P 27028 and P 31926.

\section{References}

[1] Farzamfar M, Belahcen A, Rasilo P, Clenet S, Pierquin A. Model order reduction of electrical machines with multiple inputs. IEEE Trans. Ind. Appl.. 2017; 53(4): 3355-3360. doi: 10.1109/TIA.2017.2681967.

[2] Hollaus K, Schöberl J. Some 2-D Multiscale Finite-Element Formulations for the Eddy Current Problem in Iron Laminates. IEEE Trans. Magn. 2018; 54(4): 1-4 doi: 10.1109/TMAG.2017.2777395.

[3] Klis D, Burgard S, Farle O, Dyczij-Edlinger R. A Self-Adaptive Model-Order Reduction Algorithm for Nonlinear Eddy-Current Problems Based on Quadratic-Bilinear Modeling. IEEE Trans. Magn.. 2016; 52(3): 1-4. doi: 10.1109/TMAG.2015.2487601.

[4] Sato Y, Clemens M, Igarashi H. Adaptive Subdomain Model Order Reduction With Discrete Empirical Interpolation Method for Nonlinear Magneto-Quasi-Static Problems. IEEE Trans. Magn.. 2016; 52(3): 1-4. doi: 10.1109/TMAG.2015.2489264. 
[5] Sato Y, Igarashi H. Model Reduction of Three-Dimensional Eddy Current Problems Based on the Method of Snapshots. IEEE Trans. Magn.. 2013; 49(5): 1697-1700. doi: 10.1109/TMAG.2013.2241412.

[6] Schmidthäusler D, Schöps S, Clemens M. Linear Subspace Reduction for Quasistatic Field Simulations to Accelerate Repeated Computations. IEEE Trans. Magn.. 2014; 50(2): 421-424. doi: 10.1109/TMAG.2013.2280693.

[7] Montier L, Pierquin A, Henneron T, Clénet S. Model Reduction of Three-Dimensional Eddy Current Problems Based on the Method of Snapshots. IEEE Trans. Magn.. 2017; 53(6): 1-4. doi: 10.1109/TMAG.2017.2663761.
[8] Hollaus K, Schöberl J, Schöbinger M. MSFEM and MOR to Minimize the Computational Costs of Nonlinear Eddy-Current Problems in Laminated Iron Cores. IEEE Trans. Magn.. 2020; 56(2): 1-4. doi: 10.1109/TMAG.2019.2954392.

[9] Hollaus K, Schöbinger M. A Multiscale FEM for the Eddy Current Problem with $T, \Phi$ - $\Phi$ in Laminated Conducting Media. IEEE Trans. Magn.. 2020; 56(2): 1-4. doi: 10.1109/TMAG.2019.2954480. 